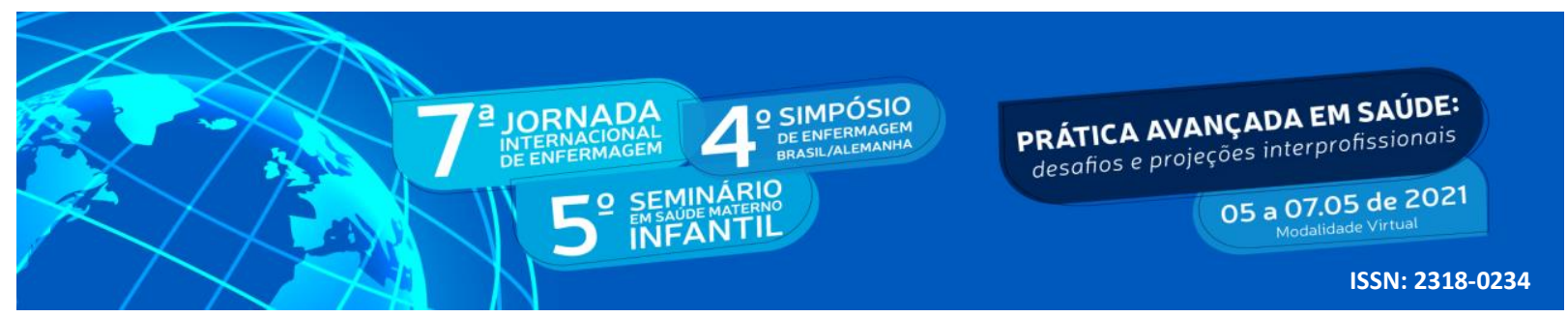

DOI: http://doi.org/10.48195/jie2021-003

\title{
IMPACTOS DA PANDEMIA DA COVID- 19 NAS NOTIFICAÇÕES DE SÍFILIS CONGÊNITA E ADQUIRIDA
}

\author{
Micheline Veras de Moura ${ }^{1}$; Aliete Cristina Gomes Dias Pedrosa da Cunha Oliveira ${ }^{2}$ Karla $^{-}$ \\ Cristina Walter ${ }^{3}$; Júlia Silva Fonseca dos Anjos ${ }^{4}$; Leila Maria Araújo Vidal ${ }^{5}$; Ana Elza Oliveira \\ de Mendonça ${ }^{6}$
}

\section{RESUMO}

A sífilis é um problema de saúde pública causado por uma infecção sistêmica pelo Treponema pallidum. Considerando que a notificação e o monitoramento das informações é de fundamental importância para a eliminação da sífilis, faz-se necessária uma investigação que forneça subsídios para planejar e definir as intervenções necessárias durante a pandemia da COVID-19. Assim, objetivou-se analisar as repercussões da pandemia nas notificações de sífilis adquirida e sífilis congênita em 2019 e 2020 . Tratase de uma pesquisa documental de abordagem quantitativa, com dados secundários do Sistema Nacional de Agravos de Notificação (SINAN). Foram notificados 34 casos de crianças com sífilis em 2019 e quatro casos em 2020, uma queda significativa de $88 \%$. Foram notificados 37 casos de sífilis adquirida em 2019 e em 2020, 0 (zero) casos, representando queda muito expressiva de 100\%. A pandemia acarretou modificações no perfil de atendimento, o monitoramento e a qualidade das notificações, o que poderá compromoter o controle e erradicação da sífilis.

Palavras-chave: COVID -19; Notificações; Sífilis adquirida; Sífilis congênita.

\begin{abstract}
Syphilis is a public health problem caused by a systemic infection by Treponema pallidum. Considering that the notification and monitoring of the information is of fundamental importance for the elimination of syphilis, an investigation is necessary that provides subsidies to plan and define the necessary interventions during the COVID-19 pandemic. The aim of this study was to analyze the repercussions of the pandemic in notifications of acquired syphilis and congenital syphilis in 2019 and 2020. This is a documentary research with a quantitative approach, with secondary data from the National System of Notifiable Diseases (SINAN). 34 cases of children with syphilis were reported in 2019 and four cases in 2020, a significant drop of $88 \%$. 37 cases of syphilis acquired in 2019 and in 2020, 0 (zero) cases were reported, representing a very significant drop of $100 \%$. The pandemic led to changes in the service
\end{abstract}

\footnotetext{
${ }^{1}$ Enfermeira Hospital Universitário Onofre Lopes. Doutoranda Universidade Coimbra. E-mail: michelineverasenf@yahoo.com;

${ }^{2}$ Professora da Escola Superior de Enfermagem de Coimbra, E-mail: aliete.cunha3@ gmail.com;

${ }^{3}$ Coordenadora do Curso de Enfermagem da IMEPAC. Doutoranda Universidade Coimbra. E-mail: karla.cris@imepac.edu.br;

${ }^{4}$ Discente do Curso de Graduação em Enfermagem da Universidade Federal do Rio Grande do Norte. E-mail: julia.sanjos18@gmail.com;

${ }^{5}$ Enfermeira Hospital Onofre Lopes. Mestre em Gestão. E-mail: leilavidal79@ hotmail.com;

${ }^{6}$ Orientador. Professora Doutora do Departamento de Enfermagem da Universidade Federal do Rio Grande do Norte. E-mail: anaelzaufrn@gmail.com
} 


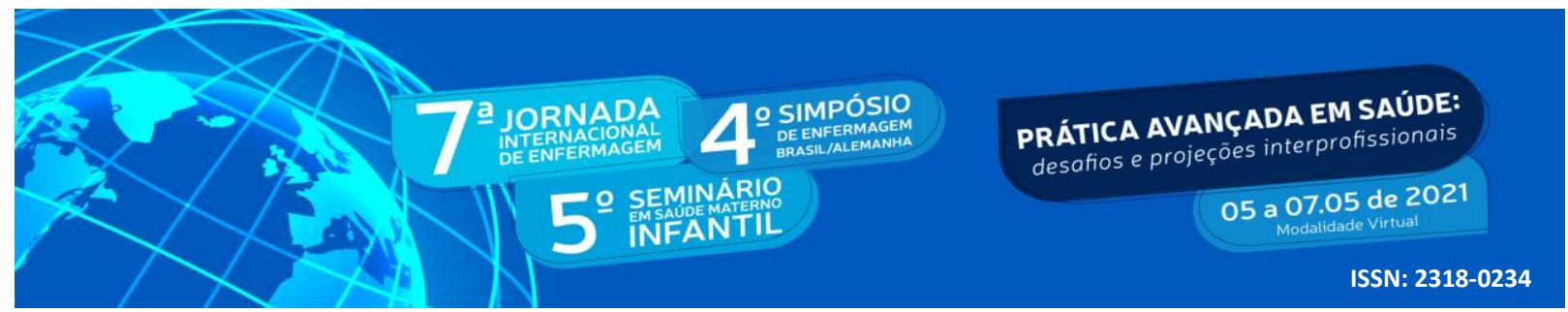

profile, monitoring and quality of notifications, which could compromise syphilis control and eradication.

Key Words: COVID-19; Notifications; Acquired syphilis; Congenital syphilis.

\section{INTRODUÇÃ̃O}

A sífilis é um problema de saúde pública e se caracteriza por uma infecção sistêmica causada pelo Treponema pallidum. Quando não tratada precocemente, pode evoluir para doença crônica com sequelas irreversíveis a longo prazo (BRASIL, 2016).

A transmissão da sífilis adquirida é por meio sexual, na área genitoanal, na quase totalidade dos casos. Na sífilis congênita, há infecção fetal via hematogênica, em qualquer fase gestacional ou estágio clínico da doença materna. Essa classificação da doença é dividida em dois períodos: a precoce, até o segundo ano de vida e a tardia que pode surgir após o segundo ano de vida (BRASIL, 2016).

A maior parte dos casos de sífilis congênita precoce é assintomática (cerca de 70\%), porém o recém-nascido pode apresentar prematuridade, baixo peso, hepatomegalia, esplenomegalia, lesões cutâneas, periostite, osteocondrite, pseudoparalisia dos membros, sofrimento respiratório com ou sem pneumonia, rinite sero-sanguinolenta, icterícia, anemia, linfadenopatia generalizada, síndrome nefrótica, convulsão e meningite, trombocitopenia, leucocitose ou leucopenia (SECRETARIA DE ESTADO DA SAÚDE- SP, 2008).

O diagnóstico de sífilis congênita é mais complexo, principalmente porque aproximadamente metade das crianças infectadas não apresenta sinais ou sintomas ao nascimento (NASCIMENTO et al., 2012). Segundo dados do Boletim Epidemiológico a sífilis adquirida no Brasil teve sua taxa de detecção aumentada de 59,1 casos por 100.000 habitantes em 2017, para 75,8 casos por 100.000 habitantes em 2018. A taxa de incidência de sífilis congênita foi de 9,0/1.000 nascidos vivos, enquanto a taxa de mortalidade foi de 8,2 /100.000 nascidos vivos persistindo um quadro epidemiológico de alta incidência em todo o país (BRASIL, 2019a ). Face à pandemia da COVID-19, a cobertura da testagem da gestante e o acompanhamento dos casos poderá ter sido mais reduzida conduzindo a medidas insuficientes para promover a eliminação da sífilis congênita. Associa-se a isso, a redução do direcionamento dos recursos 


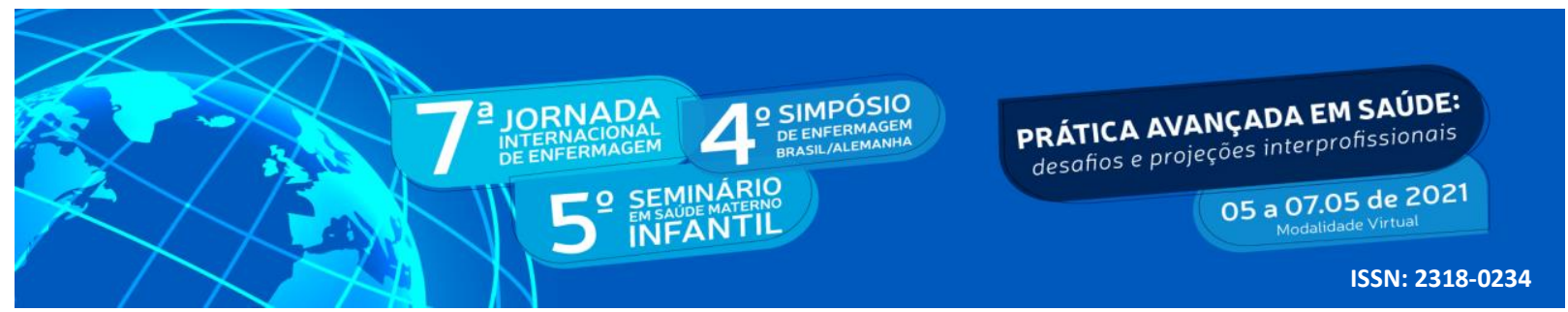

humanos disponíveis, com o objetivo de atender as demandas impostas pela pandemia como casos primordiais e emergenciais no momento do surto.

Considerando que a notificação e o monitoramento das informações é de fundamental importância para a eliminação da sífilis congênita, faz-se necessária uma investigação que forneça subsídios para planejar e definir as intervenções necessárias durante a pandemia da COVID-19.

Diante disso, consideramos analisar e comparar as notificações realizadas no Sistema Nacional de Agravos de Notificação (SINAN) das notificações de sífilis adquirida e sífilis congênita de um Hospital de Ensino, entre o período de janeiro a agosto dos anos 2019 e 2020, respectivamente, desde a data em que a Organização Mundial de Saúde declarou o surto.

\section{OBJETIVO}

Analisar as repercussões da pandemia nas notificações de sífilis adquirida e sífilis congênita em 2019 e 2020 por meio do SINAN.

\section{MATERIAIS E MÉTODO}

Estudo do tipo documental com abordagem quantitativa, realizado através dos dados da Unidade Vigilância Epidemiológica Hospitalar do Hospital Onofre Lopes (HUOL), um hospital de ensino e referência para todo estado do Rigo Grande do Norte. O local foi escolhido por ser referência no estado para sífilis e autoras deste trabalho são e foram atuantes na área e outras pesquisadoras da temática, entendo a magnitude da temática para o mundo, mas principalmente em seus locais (Brasil e Portugal) e assim contribuir com políticas para enfrentamento dessa epidemia. Os dados secundário foram coletas do período de 2019 e 2020, por meio de consultas às fichas de notificação disponíveis no banco de dados do Sistema Nacional de Agravos de Notificação (SINAN), das notificações de sífilis adquirida e sífilis congênita.

A escolha pelo local da coleta, se deu por ser um hospital de alta complexidade, referência em cardiologia, transplante e neoplasias para todo o estado do Rio Grande do Norte, Brasil. 


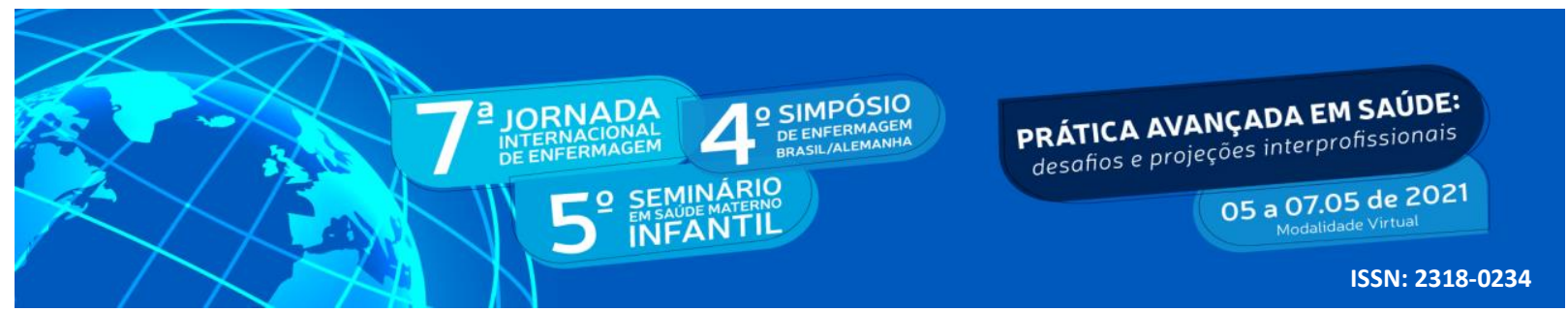

Os dados foram coletados e organizados em tabelas de frequência. Para análise dos dados foram utilizados os recursos da estatística descritiva.

Por se tratar de dados secundários o presente estudo não requer aprovação do comitê de ética em pesquisa com seres humanos conforme Resolução № 510 de 7 de abril de 2016 do Conselho Nacional de Saúde.

\section{RESULTADOS E DISCUSSÃO}

Foram analisados os dados secundários sobre sífilis congênita e sífilis adquirida coletados do SINAN do Hospital Universitário Onofre Lopes (HUOL). Os dados de sífilis congênita foram confirmados no ambulatório de infectopediatria do Hospital Universitário Onofre Lopes (HUOL), depois foram enviados em lotes para as secretarias municipal e estadual pela Unidade de Vigilância em Saúde da instituição. O ambulatório é referência para todo estado do Rio Grande do Norte.

Os dados são do período de janeiro a agosto 2019 e 2020, uma possível comparação do impacto causado pela pandemia da COVID-19 analisando as notificações de sífilis congênita e adquirida, uma infecção já negligenciada em tempos de epidemia no Brasil. Optou-se pela coleta até o mês de agosto, por serem os últimos dados disponibilizados em 2020.

Ao averiguarmos os dados sintetizados das notificações de sífilis congênita, percebemos que entre as crianças que realizaram consulta no ambulatório de infectopediatria, no período de janeiro a agosto 2019 , foram notificados 34 casos de sífilis congênita. Porém, no mês de maio ocorreu o maior número de notificações, chegando a 10 casos, representando $29 \%$ dos eventos. Do total de casos, $79 \%$ das mães realizaram pré-natal. Dessas, $41 \%$ possuem a faixa etária de 20 a 34 anos, enquanto $15 \%$ se encaixam entre 15 a 19 anos. Das gestantes, somente uma não realizou o esquema de tratamento, porém 22 tiveram tratamento inadequado, proporcional a $65 \%$ dos eventos, uma não realizou o tratamento.

Segundo o Ministério da Saúde, a penicilina é o fármaco de primeira escolha no tratamento da sífilis, com o uso exclusivo para gestantes (BRASIL, 2015). O mesmo ainda ressalta que a medicação tem uma eficácia de $98 \%$ na prevenção da sífilis congênita, agindo na fase primária, secundária e terciária. Todavia, não há relato da resistência do Treponema pallidum à penicilina. 


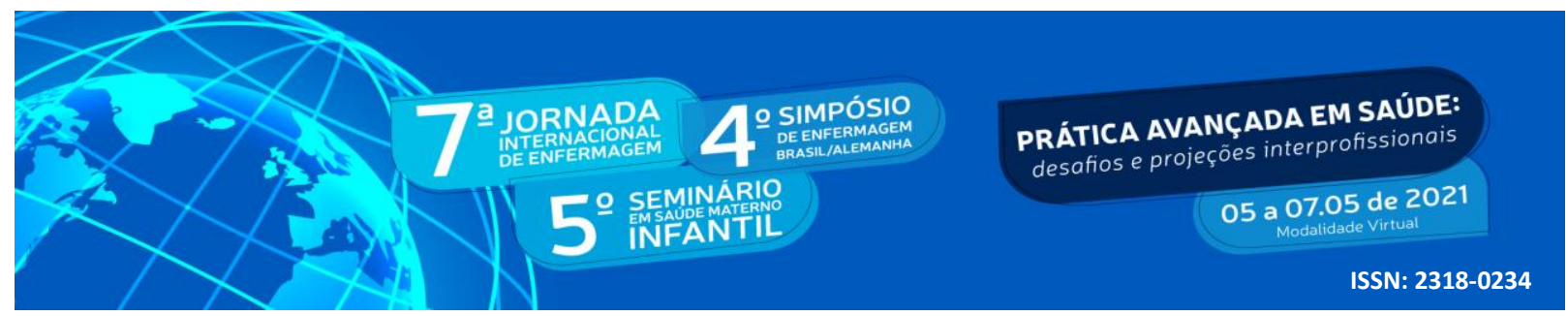

Ainda sim, essa infecção vem se mantendo com uma alta incidência nos dias de hoje, se tornando um problema de saúde pública e confronto para os serviços de saúde.Quanto ao quantitativo de dados, ressaltamos que no mês de janeiro não foi registrado nenhuma notificação. Mês esse, onde a Organização Mundial da Saúde (OMS) declarou em 30 de janeiro de 2020 que o surto da doença causada pelo novo coronavírus (COVID-19) constituiu-se como uma Emergência de Saúde Pública de Importância Internacional - o mais alto nível de alerta da Organização, conforme previsto no Regulamento Sanitário Internacional.

Em 2020, foram notificados surpreendentemente somente quatro (4) casos de sífilis congênita. Se compararmos com o ano anterior, no qual foram notificados 34 casos, a queda é significativa, pois estatisticamente temos $88 \%$ menos de notificações. Tal impacto tem magnitude, visto que o país enfrenta uma epidemia e esses resultados não condizem com a realidade e sim uma das consequências que sucedeu pela pandemia do COVID-19.

É importante destacar que, esse impacto não ocasiona consequências somente no número de notificações. Contudo, é por meio delas que o profissional atende, acolhe, testa, confirma o diagnóstico, trata, aconselha, cura e dá seguimento ao processo. Ainda vai além, quando alcança o parceiro sexual através do mapeamento.

Observou-se que uma doença já considerada negligenciada foi durante a pandemia da COVID19, ainda mais esquecida. Pois, durante os primeiros meses da pandemia, o ambulatório de infectopedia parou os atendimentos para que fossem priorizados os recursos humanos com direcionamento no atendimento dos casos de COVID-19. Porém, observou-se que com isso, indiretamente outras doenças, assim como a sífilis congênita, além de negligenciadas, vêm sendo agravadas em seu processo de monitoramento.

O Ministério da Saúde desde 2017, lançou o Projeto "Sífilis Não", o qual engloba um conjunto de ações que envolvem não apenas educação e comunicação, mas também, vigilância epidemiológica, gestão e governança, cuidado integral, e vêm sendo desenvolvidas no intitulado Projeto "Resposta Rápida a Sífilis". Porém, com a pandemia, muitas instituições e projetos foram acometidos por situações em que houve morosidade em seus projetos.

Sobre o maior número de casos ocorrer no mês de maio, não encontraram-se estudos sobre a sazonalidade da sífilis, porém, Passos (2002), relata que existem narrativas que as IST se correlacionam aos períodos carnavalescos, pelas atitudes intransigentes da população nesse período, de forma permissiva. 


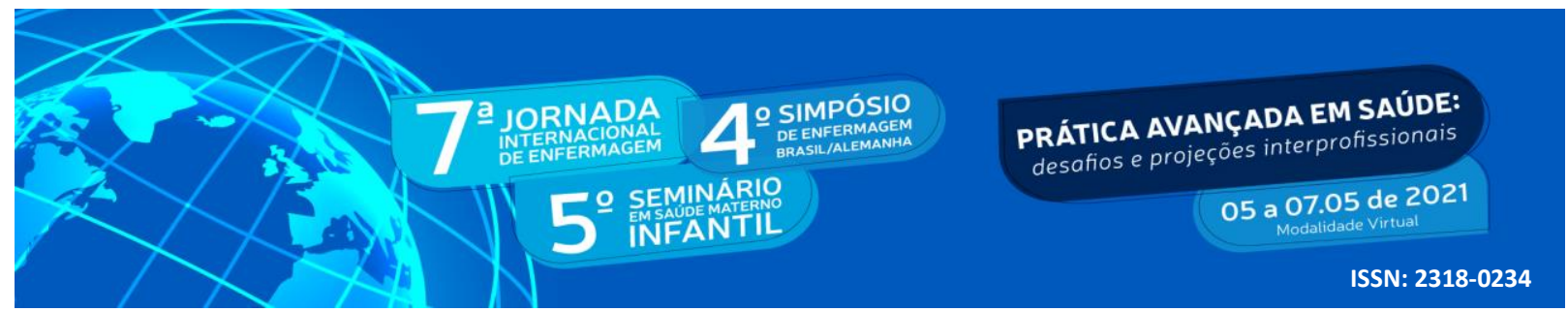

Bem mais preocupantes que a sífilis congênita, são os dados analisados sobre sífilis adquirida quando comparamos os anos de 2019 com 2020. Em 2019, foram notificados 44 casos durante o ano, e 37 até o período proposto para análise. Dos casos averiguados $46 \%$ eram do sexo masculino e 54\% do sexo feminino. Quanto à faixa etária mais acometida pela infecção, podese verificar que foram os que possuíam mais de 30 anos, correspondendo a $67 \%$ dos eventos, enquanto de 15 a 19 anos $11 \%$ e a faixa de 20 a 29 anos com $22 \%$ dos acontecimentos.

Ao analisar os dados de 2020 para averiguar o possível impacto da pandemia nas notificações de sífilis adquirida, verificou-se claramente o efeito e as consequências que trará para a população brasileira, na qual já havia sido declarada epidemia da infecção.

Abruptamente não foram registrados casos de sífilis adquirida em 2020 até o mês de agosto na instituição, representando uma queda significativa de 100\%. Não há como atribuir a queda dos casos às intervenções direcionadas para as orientações sobre sífilis, pois como foi dito anteriormente, os atendimentos ambulatoriais paralisaram para priorização das pessoas acometidas durante a pandemia.

Segundo o Ministério da Saúde as infecções sexualmente transmissíveis (IST) são consideradas um problema de saúde pública e estão entre as doenças transmissíveis mais comuns, afetando a saúde e a vida das pessoas em todo o mundo (BRASIL, 2019b). As IST têm um impacto direto sobre a saúde reprodutiva e infantil, porquanto acarretam infertilidade e complicações na gravidez e no parto, além de causar morte fetal e agravos à saúde da criança. Ressaltamos que o diagnóstico de sífilis, independente de sua classificação, é um agravo de notificação compulsória.

\section{CONCLUSÃO}

A pandemia da COVID-19 acarretou modificações no perfil de atendimento, no monitoramento e na qualidade das notificações, o que poderá impactar significativamente na vida das pessoas, além de compromoter o controle e erradicação da sífilis. Ainda são levantadas hipóteses e suposições aparentes quanto ao número de casos, pois os atendimentos ambulatoriais foram suspensos no primeiro e reiniciados no último trimestre de 2020. Segere-se que uma nova investigação seja realizada após o retorno dos atendimentos, para averiguar os agravos decorrentes da interrupção do atendimento as pessoas com sífilis durante a pandemia da COVID-19. 


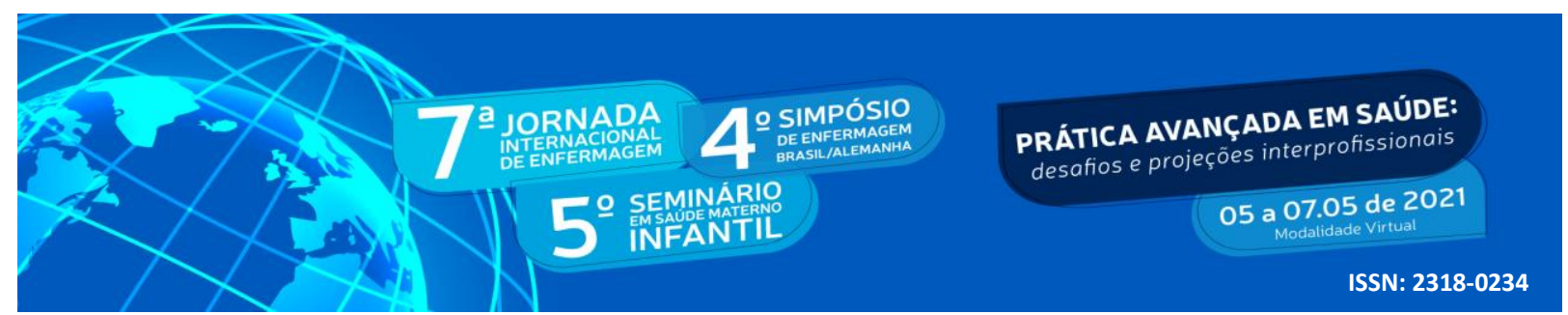

\section{REFERÊNCIAS}

BRASIL. Ministério da Saúde. Secretaria de Vigilância em Saúde Departamento de Vigilância, Prevenção e Controle das Doenças Sexualmente Transmissíveis, Aids e Hepatites Virais. Manual Técnico para Diagnóstico da Sífilis. Brasília: Ministério da Saúde; 2016.

Ministério da Saúde. Secretaria de Vigilância em Saúde. Departamento de Doenças de Condições Crônicas e Infecções Sexualmente Transmissíveis. Boletim Epidemiológico Especial Sífilis. Brasília: Ministério da Saúde ; 2019a.

Ministério da Saúde. Boletim Epidemiológico. Secretaria de Vigilância em Saúde | Ministério da Saúde Número Especial | out. 2019 b.

NASCIMENTO, M. I. et al. Gestações complicadas por sífilis materna e óbito fetal. Rev Bras Ginecol Obstet. V. 2, n 34, p. 56-62, nov. 2012.

PASSOS, M. R. L. Ocorrência de doenças sexualmente transmissíveis antes e depois do carnaval no Rio de Janeiro. DST J Bras Doenças Sex Transm. v. 14, n 38, p. 38-42. 2002.

SECRETARIA DE ESTADO DA SAÚDE - SP. Síflis congenita e sífilis gestacional . Revista de Saúde Pública; v. 4. N. 42, p. 768-72. 2008.

ORGANIZAÇÃO MUNDIAL DE SAÚDE. \{internet . [ acesso em 05 de out 2020] Disponível em: https://www.paho.org/pt/covid19 